\title{
Active gaming as a form of exercise to induce hypoalgesia
}

Christopher Carey, BS, Keith E. Naugle, PhD, Dania Aqeel, Thomas Ohlman, BA, Kelly M. Naugle, $\mathrm{PhD}$

${ }^{1}$ Department of Kinesiology, Center for Physical Activity in Wellness and Prevention, School of Physical Education and Tourism Management, Indiana University Purdue University

Indianapolis, Indiana, USA

\section{Conflicts of Interest and Source of Funding:}

The authors report no conflicts of interest related to the research or the manuscript. This study was funded by the National Institute for Fitness and Sport and School of Physical Education and Tourism Management Student Research Fund.

This is the author's manuscript of the article published in final edited form as: 


\section{ABSTRACT}

Objectives: An acute bout of moderate to vigorous exercise temporarily reduces pain sensitivity in healthy adults. Recently, active gaming has been rising in popularity as a means of light to moderate exercise and may be particularly suitable for deconditioned individuals. Whether the physical activity elicited in active games can produce a hypoalgesic effect remains unknown. The purpose of this study was to determine whether active video games can reduce pressure and heat pain sensitivity in healthy adults. We also evaluated the relationship between the physical activity elicited by the games and the magnitude of the hypoalgesic response. Methods: Twentyone healthy adults played 4 different active games on separate days, including Microsoft® Kinect Xbox One’s Fighter Within and Sports Rival’s Tennis, and Nintendo® Wii Sports’ Boxing and Tennis. Heat pain thresholds on the forearm and pressure pain thresholds (PPTs) on the trapezius and forearm were assessed immediately before and after a 15-minute active gaming or control session. Minutes spent in sedentary time and moderate to vigorous physical activity (MVPA) during active gaming were measured with an accelerometer. Results: The analyses revealed that PPTs at the forearm and trapezius significantly increased from pre to posttest following Kinect Fighter Within. PPTs at the trapezius also significantly increased from pre to posttest following Wii Boxing. The magnitude of the hypoalgesic response was significantly correlated with MVPA and sedentary time during game play. Conclusion: These results suggest that an active gaming session played at a moderate intensity is capable of temporarily reducing pain sensitivity.

Key Words: Active gaming, Exercise-induced hypoalgesia, Physical activity, Pain sensitivity 


\section{INTRODUCTION}

Exercise has shown to produce a hypoalgesic effect in healthy individuals. Specifically, studies have demonstrated that even a single session of isometric, aerobic, and dynamic resistance training can reduce the perception of experimentally induced pain. ${ }^{1}$ This alludes to the conclusion that exercise could conceivably be a modality for pain management. Thus far, most aerobic exercise-induced hypoalgesia $(\mathrm{EIH})$ research has focused on traditional modes of cardiovascular exercise, such as treadmill running or cycling. Generally, vigorous aerobic exercise at a duration of at least 15-20 minutes reduces pain sensitivity, while the data on moderate aerobic exercise is mixed. ${ }^{1,2}$ However, traditional exercise of this nature might not be plausible for an individual who is deconditioned and/or those in need of pain management.

In the past decade, active gaming has emerged as an alternative mode of physical activity that can elicit a light to moderate level of physical activity while also providing entertainment. ${ }^{4-6}$ Active games, or exergaming, are video games that elicit movement from the user. Some games utilize motion tracking technology in order to track the movement of the participant and allow the participant to interact with the game with movement of his or her body. Other active games use sensors that react to motion of the remote control. This type of exercise might be particularly suitable for deconditioned individuals because gameplay can either be intensified or decreased in real time by the participant. Games also provide built in breaks during level or opponent transitions, creating a rest time for the participant. These breaks further ensure that the gameplay is not too strenuous for the participant. However, whether active gaming can produce a sufficient level of physical activity to reduce pain sensitivity and potentially be a pain management tool remains unknown. 
The primary purpose of this study was to determine whether active video games produce a hypoalgesic effect and reduce pressure and heat pain sensitivity in healthy adults compared to a control condition. Past research indicates that the movement elicited by active games varies widely between games. ${ }^{7,8}$ Thus, we tested four different active video games from two different gaming systems including the Nintendo ${ }^{\circledR}$ Wii (an older and more widely used gaming system) and the Microsoft® ${ }^{\circledR}$ Kinect X-Box One (a newer system with updated technology). Active video games that cause more movement stimulate greater energy expenditure, with specifically hip movement being the best predictor of these responses. ${ }^{7,8}$ Therefore, the current study also assessed the level of whole-body sedentary time, light physical activity, and moderate to vigorous physical activity with an accelerometer worn on the hip. The secondary purpose was to determine the relationship between the physical activity behavior elicited by the games and the magnitude of the hypoalgesic response.

\section{METHODS}

\section{Participants}

Twenty-one healthy adults (7 males; age range 18 -32 years old; average age=22.8 \pm 4.5 years) completed this study. All participants were recruited from the university campus, right-handed, and had an average body mass index of $25.7 \pm 4.8$ (range $19.5-35.9$ ). During the first visit, participants were screened with the Physical Activity Readiness Questionnaire (PAR-Q) for any potential health conditions that would prohibit them from participating in physical activity. ${ }^{9}$ Participants were also instructed to refrain from smoking, or consuming alcohol or caffeine 24 hours prior to testing, refrain from strenuous exercise $>12$ hours prior to testing, and fast 1-hour prior to each experimental session. Participants also reported no ongoing pain conditions and no medications on days of testing prior to the testing sessions. Participants completed one 
screening/control session and four experimental sessions. The data reported here, while not published elsewhere, are part of a larger study examining the energy cost of active gaming. A power analysis using G Power 3.1.5. was used to estimate the sample size needed for detecting a significant change in pain outcome measures from the pretest (prior to active gaming) to the posttest (immediately after active gaming session). With a significance level of 0.05 , power at 0.80, a 0.5 correlation among repeated measures, the power analysis indicated that a minimum sample size of 21 subjects was needed to detect an effect size of 0.40 (moderate effect).

\section{Screening/Control Session}

All participants reviewed and signed an Informed Consent Form approved by the Institutional Review Board of Indiana University. Those individuals who sign the Informed Consent Form completed the PAR-Q and height and weight measurements. Participants were then familiarized with the pain assessments and then completed the control condition pain assessments. For the familiarization of the pain tests, participants received detailed instructions for each test and then completed three practice trials for the pressure pain thresholds and three practice trials for the heat pain thresholds on the right dorsal forearm. After a 10-minute rest period, the control condition pain assessments began. Specifically, pressure and heat pain thresholds (Described below) were assessed before and after 15 minutes of quiet rest. Lastly, participants were familiarized with the active game systems and played each active game for 10 minutes.

\section{Experimental Sessions}

All participants completed all of the experimental sessions. The order of the experimental sessions was randomized and took place on separate days. During each experimental session, participants played only one of the following games: Nintendo ${ }^{\circledR}$ Wii Sports Boxing (Wii 
Boxing), Nintendo ${ }^{\circledR}$ Wii Sports Tennis (Wii Tennis), Microsoft ${ }^{\circledR}$ X-Box One Kinect’s Sports Rival's Tennis (Kinect Tennis), or Microsoft ${ }^{\circledR} X-B o x$ One Kinect's Fighter Within (Kinect Fighter Within). A description of these games is listed in Table 1. Each game was played for two 15-minute periods with a 15-minute rest interval between each period. During period 1, participants were instructed to play at a self-selected intensity. Past research has shown that when participants play active games at a self-selected intensity, the amount of movement during games can vary widely between participants. ${ }^{6}$ Thus, during period 2, participants were instructed to try to maximize movement during game play. To avoid the effects of multiple repeated pain testing, the heat and pressure pain thresholds were only taken immediately before and after one period of game play during each session. Specifically, the pain assessments were taken pre and post period 1 (self-selected intensity) for Wii Tennis and Kinect Tennis, and pre (i.e., immediately before period 2) and post period 2 (maximize movement) for Kinect Fighter Within and Wii Boxing. This strategy allowed a wide range of physical activity intensities to be assessed during active game play, as a goal of this study was to determine if the amount and level of physical activity elicited by the active games were associated with changes in pain sensitivity. During game play, time spent in light, moderate to vigorous physical activity and sedentary time were measured using an accelerometer worn on the hip.

\section{Pain Assessments}

The pain assessments were conducted in the following order for every participant: two trials of pressure pain thresholds (PPTs) on the left ventral forearm, two trials of PPTs on the right trapezius muscle, and three trials of heat pain thresholds (HPTs) on the left ventral forearm. Assessments were conducted immediately before and after one 15-minute active gaming period during each session. The specific details for the PPTs and HPTs are described below. 
Pressure Pain thresholds (PPT). A handheld, clinical grade pressure algometer (Wagner Instruments, Greenwich, CT) was used for the mechanical procedures. The tip of the algometer consisted of a rubber flat $1.0 \mathrm{~cm}^{2}$ probe. The experimenter applied a constant rate of pressure at an approximate rate of $.5 \mathrm{~kg} / \mathrm{s}$ to either the left ventral forearm (approximately $8 \mathrm{~cm}$ distal to the elbow) or the upper right trapezius muscle (posterior to the clavicle). Participants were instructed to respond when the pressure sensation first became painful, at which time the algometer was removed and the amount of pressure in kg was recorded. The average PPT was calculated for each site pre and post each active game.

Heat Pain thresholds (HPT). Heat stimuli were delivered with a 30 x 30mm thermode (TSA II Neurosensory Analyzer; Medoc, Ltd, Ramat Yishai, Israel) placed and held on the participant's ventral forearm by the experimenter during testing. The thermode temperature increased from a baseline temperature of $32^{\circ} \mathrm{C}$ with a rise rate of $0.5^{\circ} \mathrm{C} / \mathrm{s}$. Participants were instructed to press a button when they first felt the transition from heat to the sensation of heat pain, at which time the trial stopped. Then, the temperature of the thermode was recorded. The average HPT was calculated for the three trials pre and post each active game.

Reliability of Pain Measures

We conducted interclass correlation coefficients (ICCs) on the pretests for each pain measure to determine the session-by-session reliability of these measures. The ICCs indicated excellent reliability for all pain measures: pretest PPT on the arm ICC $=0.905$; pretest PPT on the trapezius ICC=0.892; pretest HPT on the arm ICC=.969.

\section{Accelerometer Measurements}

An Actigraph GT3X+ accelerometer (The Actigraph Inc. Pensacola, FL) was worn on the hip during all sessions of active game play. The Actigraph is a small lightweight tri-axial 
accelerometer that is designed to detect tri-axial accelerations in the range of 0.05-2 G. Output from the ActiGraph was in the form of step counts, body positions and activity counts for a specific time period. Data were captured in 1-second epochs. The accelerometer data used for analyses was calculated from minutes 2 through 14 (12 minutes total) of each 15-minute active gaming period. Activity count cut-points to determine the amount of time a participant spent in sedentary, light, or moderate to vigorous activity was defined as $<100$ counts/minute (sedentary), 100-1951 counts/minute (Light physical activity), and >1951 counts/minute (moderate to vigorous activity), respectively. ${ }^{10}$ Accelerometer data are reported in minutes.

\section{Data Analysis}

Descriptives were calculated for minutes spent in sedentary time, light physical activity, MVPA, and moderate and vigorous physical activity separately for each active game. Repeated measures ANOVAs were conducted on sedentary minutes, light physical activity, moderate physical activity, and vigorous physical activity to determine differences in each type of physical activity across the games. The data for PPTs at the forearm and trapezius and HPTs were analyzed with 5(Condition: Kinect Fighter Within, Kinect Tennis, Wii Boxing, Wii Tennis, Control) $\times 2$ (Time: pre, post active game) repeated measures ANOVAs. Post-hoc comparisons were made with Tukey’s HSD procedure. Bivariate correlations were also conducted to determine the relationship between light physical activity, MVPA, and sedentary time with the magnitude of exercise-induced hypoalgesic responses, regardless of active game. For each active game, the exercise-induced hypoalgesic response for each pain test was calculated by subtracting the pretest score from the posttest score. A level of $p \leq .05$ was used for all statistical analyses.

\section{RESULTS}

\section{Descriptive Characteristics}


The accelerometer data are presented in Table 2 along with the p-values for the repeated measures ANOVA. The data indicated that whole-body movement of participants during game play only reached a moderate to vigorous intensity level during Kinect Fighter Within (moderate $=4.3 \pm 3.0$ minutes; vigorous $=1.6 \pm 2.0$ minutes) and Wii Boxing (moderate $=3.4 \pm 3.4$ minutes; vigorous=2.1 \pm 3.2 minutes). Accordingly, the ANOVAs indicated significantly higher moderate and vigorous activity in the Fighter Within and Wii Boxing games compared to the Tennis games. Participants also experienced significantly less light physical activity while playing Wii Tennis compared to all other games. All games included whole-body sedentary time ranging from Wii Tennis (self-selected intensity period) with almost 10 minutes of sedentary time to Kinect Fighter Within (maximize movement period) with just under 3 minutes of sedentary time. Sedentary time was significantly less during the Fighter Within and Wii Boxing games compared to the Tennis games.

\section{PPTs on the Forearm}

Figure 1a shows the means and standard errors of the pre- and posttests for each condition. The repeated measures ANOVA on the PPTs on the forearm revealed a significant condition $\times$ time interaction, $\mathrm{p}=.03$. Pressure pain thresholds on the forearm significantly decreased from pre to post-test during the control condition (Cohen’s $d=-0.58$ ). Pressure pain thresholds significantly increased from pre to post-test following Kinect Fighter Within (Cohen’s $d=0.41$ ). Additionally, PPTs were significantly greater during the post-test following all active games compared to PPT’s during the control condition. The main effects were not significant, p’s> .05.

\section{PPTs on the Trapezius}


Figure $1 \mathrm{~b}$ shows the means and standard errors of the pre- and posttests for each condition. The repeated measures ANOVA revealed a significant effect of time $(p=.016)$ which was superseded by a significant condition $\times$ time interaction, $p=.028$. Pressure pain thresholds on the trapezius significantly increased from pre to post-test following Kinect Fighter Within (Cohen’s $d=0.44)$ and Wii Boxing (Cohen’s $d=0.42$ ).

\section{HPTs on the Forearm}

Figure 1c shows the means and standard errors of the pre- and posttests for each condition. The analysis revealed a significant main effect of condition, $\mathrm{p}<.001$. HPTs were lower in the control condition compared to the active game conditions. A significant main effect of time $(\mathrm{p}<.001)$ also revealed that HPTs increased from pre-test to post-test across all conditions.

\section{Bivariate Correlations between EIH and Time spent in Light Physical activity, MVPA, or}

\section{Sedentary Time}

The bivariate correlations among EIH for each pain test and physical activity variable are presented in Table 3. Exercise-induced hypoalgesia measured with PPTs at the forearm and trapezius were positively correlated with minutes spent in MVPA and negatively associated with sedentary time.

\section{DISCUSSION}

Prior research has established that an acute bout of traditional moderate to vigorous aerobic exercise can reduce pain sensitivity in healthy individuals. ${ }^{1}$ However, less is known regarding whether alternative modes of physical activity that might be more appealing and plausible for deconditioned individuals can also produce a hypoalgesic effect. The current study was the first to evaluate whether a single session of active gaming can reduce pain sensitivity. 
Active gaming provides an avenue for participants to be physically active in their home while also being entertained. We discovered that the two games eliciting the greatest moderate to vigorous physical activity reduced pressure pain sensitivity, while the two games eliciting minimal MVPA had no impact on pain perception.

Kinect Fighter Within increased PPTs on the forearm and trapezius, with a moderate effect size. This coincided with the fact that participants displayed the most moderate to vigorous physical activity (5.9 \pm 4.1 minutes out of 12 minutes) while playing this game, which involves the upper and lower body due to the kicking and punching involved in game play. Importantly, the hypoalgesic effect was still present even though approximately half of game play was spent in either whole body sedentary or light physical activity levels (2.9 \pm 2.7 and 3.2 \pm 1.6 minutes, respectively), even when the participants were instructed to maximize movement.

Wii boxing reduced pressure pain sensitivity on the trapezius, but not the forearm. Similar to Kinect Fighter Within, participants spent just under half of game play in whole body moderate to vigorous activity (5.5 \pm 4.4 minutes out of 12 minutes). Prior EIH research has widely shown that short-term ( 2-5 minutes), high intensity isometric exercise exerts generalized hypoalgesic effects (i.e., pain reduction on exercised and non-exercised parts of the body). ${ }^{11,12,13}$ In the current study, a lack of sustained exercise at higher intensities may have contributed to the lack of a larger generalized hypoalgesic effect induced by active gaming. Indeed, the 15 minutes of game play included bouts of MVPA interspersed with bouts of light physical activity and even at times no physical activity.

The observed exercise-induced hypoalgesic effects on the trapezius but not the forearm during Wii boxing could have been caused by differential activation of local pain inhibitory effects at the trapezius versus forearm. Past research suggests that EIH post exercise may be 
produced by local and central pain inhibitory effects and effects may be stronger when local and central effects are combined. ${ }^{13,14}$ Indeed, the movement elicited during Wii boxing is primarily arm punches which involve the trapezius muscle more than muscles of the forearm. However, it should be noted that a primary movement of Kinect Fighter Within is also arm punches. Additionally, PPT at the trapezius was measured on the dominant side, whereas PPT on the forearm was taken on the non-dominant side. While only speculation, the dominant arm was likely exercised (i.e., threw more punches) more than the non-dominant arm during game play. As such, the trapezius muscle of dominant arm could have been exposed to greater activation of localized pain inhibitory mechanisms during Wii Boxing compared to the forearm muscles of the non-dominant arm.

These results also differ from those seen in previous studies conducted by Hoffman et al. and Naugle et al. where the participants only showed an exercise induced hypoalgesic effect for PPTs after sustained vigorous activity ( 20-30 minutes) but not after sustained moderate intensity or shorter vigorous bouts (10 minutes). ${ }^{2,3}$ In the current study, EIH effects were produced within a shorter time frame and at a lower intensity. The location of the pain testing and/or the type of exercise training could have contributed to the contrasting results. The pain testing in the Hoffman and Naugle studies were conducted on body sites not involved in the exercise (i.e., index finger and forearm), where as in the current study the pain tests were conducted on the forearm and trapezius which were likely involved in active game play. Additionally, complete comparisons with these studies is difficult because exercise intensity was measured with different methods across studies (Heart rate/ $\mathrm{VO}_{2}$ max in Naugle and Hoffman studies vs. accelerometry in current study). Future research needs to determine the importance of exercising the tested body part for changes in pain sensitivity during active gaming. 
Kinect Tennis and Wii Tennis did not reduce pain sensitivity on any measure. This result is not surprising given that the tennis games produced negligible whole-body moderate to vigorous physical activity and only approximately two minutes of whole-body light physical activity. Our exercise intensity data are in agreement with other active gaming studies showing that Wii Tennis is a light intensity activity when played at a self-selected intensity. ${ }^{5,6}$ The lack of an EIH effect during the tennis games combined with our correlational data highlight the importance of moderate to vigorous physical activity for EIH following aerobic exercise.

Interestingly, our results indicated that heat pain sensitivity decreased across all conditions. Thus, while HPTs increased following active gaming, it also increased following 15 minutes of quiet rest. Thus, pain reduction was likely caused by repeated pain testing rather than by active gaming. Similarly, Vaegter and colleagues recently found comparable increases in HPTs between isometric exercise and quiet rest conditions. ${ }^{15}$ Our results are also in line with previous studies showing no effect of aerobic exercise on HPTs, and that EIH responses are not consistent across experimental pain tests, demonstrating the importance of multi-modal pain assessment when studying EIH. . $^{215-18}$

A few limitations of this study should be noted. First, only young to middle-age healthy adults were tested and these results may not generalize to older adults or individuals with chronic pain. Secondly, participants played each active game for two 15-minute periods (i.e., selfselected intensity period and maximize movement period) and the current study did not measure pain thresholds before and after each period. Consequently, it cannot be determined whether Fighter Within or Wii Boxing played at a self-selected intensity would produce EIH. Conversely, the Tennis games played with instructions to maximize movement may have produced analgesic effects. Likely, the primary factor as to whether an active game exerts an analgesic effect is the 
intensity level reached during game play. Third, while the order of the experimental active gaming sessions were randomized across participants, the control session was always conducted first. Thus, we cannot rule out the possibility of order effects between the control session and the experimental sessions. Fourth, we based our assessment of exercise intensity on accelerometer data captured at the hip, which would not have captured all bodily movement (i.e., upper limbs).

\section{Clinical Implications and Future Directions}

Exercise can cause an acute hypoalgesic effect that could be beneficial for those suffering from different forms of chronic pain. However, many of these individuals are deconditioned and unable to exercise at sustained higher intensities because of their conditions. Additionally, several studies suggest that vigorous aerobic exercise fails to produce a hypoalgesic effect in individuals with central pain inhibitory deficiencies, such as fibromyalgia patients and chronic fatigue syndrome. ${ }^{16,19,20}$ We provide preliminary evidence that active gaming may have value as an exercise tool for pain management. Indeed, we showed that active gaming can produce an exercise-induced hypoalgesic effect without long bouts of strenuous activity in healthy adults. Notably, both Kinect Fighter Within and Wii Boxing reduced pain sensitivity even though on average $25 \%$ of the time assessed during game play was spent in whole-body sedentary time. Furthermore, in individuals with chronic pain the act of interacting with the game could also enhance compliance with exercise therapy and serve as a pleasant distraction from pain symptoms. In support of this notion, Mortensen et al. recently revealed in an observational study that individuals with fibromyalgia reported that active games provided a manageable form of exercise and distraction from pain sensations while playing. ${ }^{21}$ Thus, active gaming may be a viable option for deconditioned individuals and those suffering from chronic pain. Additional research should examine active gaming in chronic pain populations to test its efficacy as a 
Active gaming and pain sensitivity 15

cardiovascular and pain management tool. If deemed effective, active gaming could pose as an inexpensive, pragmatic tool to aid in the rehabilitation of individuals suffering from chronic pain. 
Active gaming and pain sensitivity 16

\section{Author Disclosure Statement}

No competing financial interests exist. 


\section{REFERENCES}

1. Naugle KM, Fillingim RB, Riley JL. A meta-analytic review of the hypoalgesic effects of exercise. The Journal of pain 2012;13(12):1139-1150.

2. Naugle KM, Naugle KE, Fillingim RB, Samuels B, Riley JL III. Intensity thresholds for aerobic exercise-induced hypoalgesia. Med Sci Sports Exerc 2014;46:817-25.

3. Hoffman MD, Shepanski M, Ruble S, Valic Z, Buckwalter J, Clifford P. Intensity and duration threshold for aerobic exercise-induced analgesia to pressure pain. Arch Phys Med Rehabil 2004;85:1183-1187.

4. O’Donovan C, Hirsch E, Holohan E, McBride I, McManus R, Hussey J. Energy expended playing Xbox Kinect ${ }^{\mathrm{TM}}$ and Wii ${ }^{\mathrm{TM}}$ games: a preliminary study comparing single and multiplayer modes. Physiotherapy 2012;98(3):224-229.

5. O’Donovan C, Hussey J. Active video games as a form of exercise and the effect of gaming experience: a preliminary study in healthy young adults. Physiotherapy 2012;98(3):205-210.

6. Naugle KE, Naugle KM, Wikstrom EA. Cardiovascular and affective outcomes of active gaming; using the Nintendo Wii as a cardiovascular training tool. J Strength Cond Res 2014;28(2):443-51.

7. Graves LE, Ridgers ND, Stratton G. The contribution of upper limb and total body movement to adolescents’ energy expenditure whilst playing Nintendo Wii. European Journal of Applied Physiology 2008;104(4):617-623.

8. Jordan M, Donne B, Fletcher D. Only lower limb controlled interactive computer gaming enables an effective increase in energy expenditure. European Journal of Applied Physiology 2011;111(7):1465-1472. 
9. Thomas S, Reading J, Shephard RJ. Revision of the Physical Activity Readiness Questionnaire (PAR-Q). Canadian Journal of Sport Sciences 1992;17(4):338-345.

10. Freedson PS, Melanson E, Sirard J. Calibration of the Computer Science and Applications, Inc. accelerometer. Medicine and Science in Sports and Exercise 1998; 30:777-781.

11. Koltyn K, Umeda M. Contralateral attenuation of pain after short-duration submaximal isometric exercise. J Pain 2007;8:887-92.

12. Hoeger Bement M, Dicapo J, Rasiarmos R, Hunter S. Dose response of isometric contractions on pain perception in healthy adults. Med Sci Sports Exerc 2008;40:1880-89.

13. Kosek E, Lundberg L. Segmental and plurisegmental modulation of pressure pain thresholds during static muscle contractions in healthy individuals. Eur J Pain 2003;7:251-5

14. Black CD, Tynes BK, Gondlach AR, Waddell DE. Local and Generalized Endogenous Pain Modulation in Healthy Men: Effects of Exercise and Exercise-Induced Muscle Damage. Pain Med 2016; epub ahead of print.

15. Vaegter HB, Hoeger Bement M, Madsen AB, Fridriksson J, Dasa M, Graven-Nielsen T. Exercise increases pressure pain tolerance but not pressure and heat pain thresholds in healthy young men. Eur J Pain 2016; epub ahead of print.

16. Cook DB, Stegner AJ, Ellingson LD. Exercise alters pain sensitivity in Gulf War veterans with chronic musculoskeletal pain. J Pain 2010;11:764-772.

17. Kodesh E, Weissman-Fogel I. Exercise-induced hypoalgesia - interval versus continuous mode. Appl Physiol Nutr Metab 2014;39(7):829-34.

18. Naugle KM, Naugle KE, Fillingim RB, Riley JL $3^{\text {rd }}$. Isometric exercise as a test of pain modulation: effects of experimental pain test, psychological variables, and sex. Pain Med 2014;15:692-701. 
19. Meeus M, Roussel N, Truijen S, Nijs J. Reduced pressure pain thresholds in response to exercise in Chronic Fatigue Syndrome but not in Chronic Low Back Pain: An experimental study. J Rehab Med 2010;42:884-890.

20. Vierck C, Staud R, Price D, Cannon R, Mauderli A, Martin A. The effect of maximal exercise on temporal summation of second pain (windup) in patients with Fibromyalgia Syndrome. J Pain 2001;2:334-244.

21. Mortensen J, Kristensen LQ, Brooks EP, Brooks AL. Women with fibromyalgia’s experience with three motion-controlled video game consoles and indicators of symptom severity and performance of activities of daily living. Disability and Rehabilitation: Assistive Technology 2015;10(1):61-66. 


\section{FIGURE LEGENDS}

Figure 1. Means and Standard Errors for the pressure pain thresholds (PPT's) on the forearm (A), PPT's on the trapezius (B), and heat pain thresholds (HPT’s) on the forearm pre and post each condition (C). For HPT's a significant main effect of time was found, in which HPT's increased significantly from pre to posttest regardless of condition. *=significant difference at the $\mathrm{p}<.05$ level. $\mathrm{N}=21$ participants. Note: the start of the $\mathrm{Y}$-axis for each Figure does not equal absolute zero. 


\section{Corresponding Author:}

Kelly M. Naugle

Indiana University Purdue University Indianapolis

901 W. New York St.

Indianapolis, IN 46202

Phone: 317-274-0601

Fax: 317-278-2041

Email: kmnaugle@iupui.edu 
Table 1. Description of Active Games.

\begin{tabular}{|l|l|}
\hline Game & \multicolumn{1}{|c|}{ Description } \\
\hline Wii Boxing & Wii Sports upper extremity game, which uses both upper extremities with \\
\hline Wii Tennis & Wii Sports upper extremity game, which uses the dominant arm with a hand \\
controller to mimic tennis movements.
\end{tabular}


Table 2. Minutes (Mean \pm Standard Deviation) of Whole-body Light Physical Activity, Moderate and Vigorous Physical Activity, and Sedentary Time for each Active Game.

\begin{tabular}{lcccc}
\hline $\mathrm{N}=21$ & Sedentary & Light PA & Moderate PA & Vigorous PA \\
\hline Kinect Fighter Within^ & $2.9 \pm 2.7$ & $3.2 \pm 1.6$ & $4.3 \pm 3.0$ & $1.6 \pm 2.0$ \\
Kinect Tennis* & $9.2 \pm 2.5$ & $2.2 \pm 1.8$ & $0.6 \pm 1.1$ & $0.0 \pm 0.0$ \\
Wii Boxing^ & $3.1 \pm 3.5$ & $3.4 \pm 2.4$ & $3.4 \pm 3.4$ & $2.1 \pm 3.2$ \\
Wii Tennis* & $9.9 \pm 2.5$ & $1.7 \pm 1.4$ & $0.4 \pm 0.2$ & $0.0 \pm 0.0$ \\
p-value & $<.01$ & .026 & $<.01$ & .035 \\
\hline
\end{tabular}

Note. PA=Physical activity; Total time measured was 12 minutes. *Games played at a selfselected intensity. ^ Games played with participants instructed to maximize movement. P-values were calculated from repeated measures ANOVA across games. 
Table 3. Bivariate Correlations between Physical Activity Variables and Magnitude of Exercise-Induced Hypoalgesia across all Active Games

\begin{tabular}{lllllll}
\hline Variable & 1 & 2 & 3 & 4 & 5 & 6 \\
\hline 1. Sedentary Minutes & 1.00 & & & & & \\
2. Light PA Minutes & $-0.53^{* *}$ & 1.00 & & & & \\
3. MVPA Minutes & $-0.84^{* *}$ & 0.06 & 1.00 & & & \\
4. EIH PPTs on forearm & $-0.26^{*}$ & -0.12 & $0.39^{* *}$ & 1.00 & & \\
5. EIH PPTs on trapezius & $-0.25^{*}$ & 0.01 & $0.33^{* *}$ & $0.29^{* *}$ & 1.00 & \\
6. EIH HPTs on forearm & 0.02 & 0.01 & -0.02 & 0.18 & 0.14 & 1.00 \\
& & & & & & \\
\hline
\end{tabular}

Note. PA=physical activity; MVPA=moderate to vigorous physical activity; EIH=exercise-

induced hypoalgesia; PPTs=pressure pain thresholds; HPTs=heat pain thresholds;

**=Correlation is significant at the 0.01 level (2-tailed); *=Correlation is significant at the 0.05

level (2-tailed). Table represents data from 21 participants, with each participant having four data points for each variable (i.e., one for each game). 
